\title{
Commentary: Decomposition of Heart Rate Variability Spectrum into a Power-Law Function and a Residual Spectrum
}

\author{
Paolo Castiglioni* \\ IRCCS Fondazione Don C. Gnocchi, Milan, Italy
}

Keywords: heart rate variability, 1/f slope, power spectral analysis, fractals, self-similarity

\section{A commentary on}

Decomposition of Heart Rate Variability Spectrum into a Power-Law Function and a Residual Spectrum

by Kuo J and Kuo C. -D. Front. Cardiovasc. Med. (2016). 3:16. doi: 10.3389/fcvm.2016.00016

\section{OPEN ACCESS}

Edited by:

Shimon Rosenheck,

Meir Medical Center, Israel

Reviewed by:

Osmar Antonio Centurion, Universidad Nacional de Asunción,

Paraguay

Javier Eduardo Banchs, Scott and White Memorial Hospital,

United States

*Correspondence:

Paolo Castiglioni

pcastiglioni@dongnocchi.it

Specialty section:

This article was submitted to

Cardiac Rhythmology,

a section of the journal

Frontiers in Cardiovascular Medicine

Received: 01 February 2018

Accepted: 26 June 2018

Published: 26 July 2018

Citation:

Castiglioni P (2018) Commentary:

Decomposition of Heart Rate Variability Spectrum into a Power-Law

Function and a Residual Spectrum.

Front. Cardiovasc. Med. 5:94.

doi: 10.3389/fcvm.2018.00094
Recently J.Kuo and C.D.Kuo published a method for decomposing the power spectrum, $P S D$, of heart rate variability (HRV) in a power-law function "displaying the fractal characteristics of the $P S D$ " and in a residual spectrum, rHRV (1). Readers of this journal should consider important methodological aspects of this approach to interpret correctly its results.

The method is based on plotting PSD vs. the frequency Frq in a log-log scale and on estimating the slope, $s$, and the intercept, $Y$, of the regression line, $r g$, fitting $\log (P S D)$ vs. $\log (F r q)$ :

$$
r g=s \times \log (F r q)+Y
$$

Then the regression line is anti-transformed to obtain the power law component, $P S D_{r g}$ :

$$
P S D_{r g}=10^{s \times \log (F r q)+Y}
$$

and the residual, $r P S D$, defined as:

$$
r P S D=\frac{P S D}{P S D_{r g}}
$$

The authors express $r P S D$ in units of the power spectrum $\left(\mathrm{ms}^{2} / \mathrm{Hz}\right.$ if the tachogram is measured in $\mathrm{ms}$ ), assuming $P S D_{r g}$ to be a dimensionless quantity. However, any regression line has the units of the data that it fits. Therefore, $r g$ in Equation (1) has the units of $\log (P S D)$ and after the antitransformation, $P S D_{r g}$ has the units of $10^{\log (P S D)}$, i.e., of $P S D$. This means that $P S D_{r g}$ is expressed in $\mathrm{ms}^{2} / \mathrm{Hz}$ and that the spectral ratio $r P S D$ in Equation (3) is a dimensionless number

This explains why $P S D_{r g}$ shows the same range of values of the original spectrum in Figure 1C of (1). This also explains the surprisingly very low values of $r P S D$ in Figure 1D and of the $r H R V$ high frequency (HF) power in Figure 2. In fact, very low values at the higher frequencies are surprising if obtained after removal from the original spectrum of a power-law component whose contribution should be minimal at the higher frequencies. But actually, the statistical comparison between powers of $H R V$ and powers of $r H R V$ in the left panels of Figure 2 has no meaning, because 
the two quantities have different units. The authors suggest in their conclusions that "the clinical meaning and significance of rHRV measures might be different from traditional $H R V$ measures": this is certainly true because the $r H R V$ measures introduced in (1) cannot be considered measures of HRV power.

A second methodological aspect deserves to be commented on. The authors fit the regression line up to the Nyquist frequency, i.e., the highest frequency of the spectrum. Starting from the first report of a " $1 / \mathrm{f}$ " trend in the HRV spectrum (2), the literature considered only frequencies lower than the low frequency (LF) band to exclude non-fractal components, like the respiratory sinus arrhythmia and the Mayer waves, from the regression fitting (3). For this reason, $P S D_{r g}$ cannot be considered the true "fractal component" of the spectrum, because the regression slope $s$ is influenced by oscillations in the LF and HF bands. International guidelines on HRV recommended estimating the " $1 / \mathrm{f}$ " trend only on frequencies $<0.04 \mathrm{~Hz}$ (4). This requires long-term recordings and the short duration of the HR series considered in this work (512 beats, i.e., less than $7 \mathrm{~min}$ ) does not allow estimating the regression slope on

\section{REFERENCES}

1. Kuo J, Kuo C-D. Decomposition of heart rate variability spectrum into a powerlaw function and a residual spectrum. Front Cardiovasc Med. (2016) 3:16. doi: $10.3389 /$ fcvm.2016.00016

2. Kobayashi M, Musha T. 1/f fluctuation of heartbeat period. IEEE Trans Biomed Eng. (1982) 29:456-7.

3. Sassi R, Cerutti S, Lombardi F, Malik M, Huikuri HV, Peng CK, et al. Advances in heart rate variability signal analysis: joint position statement by the e-Cardiology ESC Working Group and the European Heart Rhythm Association co-endorsed by the Asia Pacific Heart Rhythm Society. Europace (2015) 17:1341-53. doi: 10.1093/europace/ euv015

4. Task Force of the European Society of Cardiology and the North American Society of Pacing and Electrophysiology. Heart rate variability. Standards of measurement, physiological interpretation, and clinical use. Eur Heart J. (1996) 17:354-81.

5. Yamamoto Y, Hughson RL. Coarse-graining spectral analysis: new method for studying heart rate variability. J Appl Physiol. (1991) 71:1143-50. a sufficient number of spectral lines. It should be considered, however, that an alternative method allows extracting powerlaw fractal components even from HRV spectra estimated on similarly short data segments. This is possible by exploiting the fact that coarse graining the tachogram preserves the self-similar dynamics of HR and not the LF and HF periodic oscillations (5). The coarse-graining method is used not only to quantify the fractal component of HR even from relatively short segments of data (6), but also to better estimate the LF and HF oscillatory components by removing the underling fractal power $(7,8)$.

In conclusion, the $r P S D$ function proposed in (1) appears an interesting way to quantify deviations from the power-law trend. However, it cannot be considered a measure of spectral power, and it may be still affected by HR fractal components if the least-square fitting includes the LF band.

\section{AUTHOR CONTRIBUTIONS}

The author confirms being the sole contributor of this work and approved it for publication.

6. Fortrat JO, Sigaudo D, Hughson RL, Maillet A, Yamamoto Y, Gharib C. Effect of prolonged head-down bed rest on complex cardiovascular dynamics. Auton Neurosci. (2001) 86:192-201. doi: 10.1016/S1566-0702(00)00212-5

7. Yamamoto Y, Hoshikawa Y, Miyashita M. Effects of acute exposure to simulated altitude on heart rate variability during exercise. J Appl Physiol. (1996) 81:1223-9.

8. Butler GC, Senn BL, Floras JS. Influence of atrial natriuretic factor on heart rate variability in normal men. Am J Physiol. (1994) 267:H500-5.

Conflict of Interest Statement: The author declares that the research was conducted in the absence of any commercial or financial relationships that could be construed as a potential conflict of interest.

Copyright (c) 2018 Castiglioni. This is an open-access article distributed under the terms of the Creative Commons Attribution License (CC BY). The use, distribution or reproduction in other forums is permitted, provided the original author(s) and the copyright owner(s) are credited and that the original publication in this journal is cited, in accordance with accepted academic practice. No use, distribution or reproduction is permitted which does not comply with these terms. 